\title{
Deregulation of the NLRP3 inflammasome in hepatic parenchymal cells during liver cancer progression
}

\author{
Qing Wei ${ }^{1,5}$, Kun $\mathrm{Mu}^{2,5}$, Tao $\mathrm{Li}^{3}$, Ying Zhang ${ }^{1}$, Zhaowen Yang ${ }^{1,3}$, Xiaoqing Jia ${ }^{4}$, Wei Zhao ${ }^{1}$, Wanwan Huai ${ }^{1}$, \\ Pengbo Guo ${ }^{1}$ and Lihui Han $^{1}$
}

Hepatocellular carcinoma (HCC) is one of the most prevalent malignant tumors worldwide, and it is always the consequence of chronic hepatitis and liver cirrhosis. The nucleotide-binding domain, leucine-rich family (NLR), pyrin-containing 3 (NLRP3) inflammasome has been shown to orchestrate multiple innate and adaptive immune responses. However, little is known about its role in cancer. This study was performed to investigate the role of the NLRP3 inflammasome in the development and progression of HCC. The expression of NLRP3 inflammasome components was analyzed in HCC tissues and corresponding non-cancerous liver tissues at both the mRNA and protein levels. Our data demonstrate that the expression of all of the NLRP3 inflammasome components was either completely lost or significantly downregulated in human HCC, and that the deficiency correlated significantly with advanced stages and poor pathological differentiation. In addition, our data provide an overview of the expression of NLRP3 inflammasome components in the multi-stage development of HCC and indicate a surprising link between deregulation of the NLRP3 inflammasome molecular platform and HCC progression. In conclusion, this study presents a dynamic expression pattern of NLRP3 inflammasome components in multi-stage hepatocarcinogenesis and demonstrates that deregulated expression of the inflammasome is involved in HCC progression.

Laboratory Investigation (2014) 94, 52-62; doi:10.1038/labinvest.2013.126; published online 28 October 2013

KEYWORDS: carcinogenesis; hepatocellular carcinoma; inflammasome; NLRP3; parenchymal cell

Inflammasomes are intracellular multiprotein complexes that act as platforms for the maturation and secretion of the proinflammatory cytokines interleukin (IL)-1 $\beta$ and IL-18. The nucleotide-binding domain, leucine-rich family (NLR), pyrin-containing 3 (NLRP3) inflammasome is currently the most characterized inflammasome, and it consists of a scaffold protein (NLRP3), an apoptosis-associated speck-like protein containing a caspase-recruitment domain (ASC) adaptor, and caspase 1. Upon activation of the NLRP3 inflammasome, pro-caspase 1 is recruited to the platform and then autocatalytically cleaved to its active form, which subsequently cleaves pro-IL- $1 \beta$ in the cytoplasm into mature IL- $1 \beta$ and allows its secretion, finally resulting in inflammation. ${ }^{1,2}$ Therefore, the primary role of the inflammasome is to direct inflammation when the host is in danger. ${ }^{2-5}$ Over the past decades, many studies have focused on the role that the NLRP3 inflammasome has in the innate immune system as a sensor of pathogens and danger signals, and its activation mechanism has been widely investigated. However, whether the NLRP3 inflammasome is involved in cancer progression has not been well studied. Hepatocellular carcinoma (HCC) is the most common primary malignant tumor of the liver, and it is the third leading cause of cancer death worldwide. ${ }^{6}$ In this study, we investigated the role that the NLRP3 inflammasome has in the progression of HCC.

The formation and function of the NLRP3 inflammasome in myeloid immune cells in normal and pathogenic states have been well characterized. However, increasing evidence shows that inflammasomes also assemble in non-myeloid cells and have roles in many diseases. ${ }^{7,8}$ As hepatic parenchymal cells are essential functional elements and constitute the dominant population of the liver, they contribute significantly to the

\footnotetext{
Department of Immunology, Shandong University School of Medicine, Jinan, China; ${ }^{2}$ Department of Pathology, Shandong University School of Medicine, Jinan, China; ${ }^{3}$ Department of Gastroenterology, Provincial Hospital Affiliated with Shandong University, Jinan, China and ${ }^{4}$ Department of Gastroenterology, Shandong University Qilu Hospital, Jinan, China

Correspondence: Dr LH Han, MD, PhD, Department of Immunology, Shandong University School of Medicine, 44 Wenhua Xi Road, Jinan, Shandong 250012 China. E-Mail: hanlihui@sdu.edu.cn

${ }^{5}$ These authors contributed equally to this work.

Received 7 July 2013; revised 22 September 2013; accepted 4 October 2013
} 
pathogenesis of chronic liver diseases such as liver cancer. ${ }^{9,10}$ Given the growing body of evidence of a diverse, multifaceted role of the NLRP3 inflammasome in non-myeloid epithelial cells, ${ }^{7,8,11}$ it is plausible that it is involved in hepatic malignant transformation and HCC progression. It remains unclear whether the NLRP3 inflammasome functions detrimentally or beneficially in hepatic parenchymal cells during liver cancer progression, and in this study, we therefore investigated its involvement.

A review of the literature showed that most studies that have attempted to determine the role of the inflammasome in disease progression were conducted using specific gene knockout animal models ${ }^{11,12}$ that could mimic the pathological situation to a certain degree; however, these models may not precisely reflect the situation in patients. To our knowledge, this is the first study assessing the role of the NLRP3 inflammasome in the clinic in liver cancer patients. Our data surprisingly suggest that the NLRP 3 inflammasome is significantly downregulated in liver cancer tissues when compared with non-cancerous liver tissues and that patients with more advanced HCC have lower expression levels of the NLRP3 inflammasome components, indicating that the downregulation of the multiprotein NLRP3 inflammasome platform is involved in HCC progression. These findings suggest that in addition to its well-recognized role as an inducer of immunity and inflammation, the NLRP3 inflammasome has a role in cancer development.

\section{MATERIALS AND METHODS \\ Patients, Specimens, and Tissue Microarray Construction}

Matched pairs of primary HCC samples and adjacent noncancerous liver tissues were used to construct a tissue microarray (Shanghai Biochip Co., Ltd., Shanghai, China). Two cores were taken from each formalin-fixed, paraffinembedded sample using punches (1.5 $\mathrm{mm}$ in dimension) from the non-necrotic areas of cancer foci and corresponding peritumoral non-cancerous liver tissues. In total, 128 HCC patients, including 111 males and 17 females, with cores containing cancerous and corresponding non-cancerous tissue, were included for immunohistochemistry (IHC) analysis (Table 1). To further elucidate alterations in the expression of NLRP3 inflammasome components in HCC patients, 9 normal liver tissues were also used for IHC analysis as controls. The normal liver controls were obtained from surgical specimens obtained from patients undergoing liver resection, in whom all causes of liver disease had been excluded (ie, medication, alcohol, chronic viral hepatitis, autoimmune processes, and metabolic disease). The normal liver controls were carefully reviewed by two liver pathologists and considered normal: no portal or lobular inflammation, absence of necrosis and fibrosis, no ballooning, and no cholestasis or bile duct lesions.

To validate the results of the IHC analysis of the cancerous liver tissues, paired pathology-proven fresh HCC tissues with corresponding non-cancerous liver tissues were obtained from another cohort of 24 HCC patients, who underwent curative resection in the Department of Hepatobiliary Surgery of the Provincial Hospital Affiliated with Shandong University. No patients received chemotherapy or radiotherapy before surgical resection. Cell differentiation based on tumor grading was determined by the Edmondson Grading System, and clinical stages were classified according to the International Union against Cancer TNM classification system.

The third cohort of chronic hepatitis patients was recruited from the Department of Gastroenterology of the Provincial Hospital Affiliated with Shandong University. Liver biopsy specimens $(\sim 1.5 \mathrm{~mm}$ in diameter and nearly $1.5 \mathrm{~cm}$ long $)$ were obtained using a percutaneous 14-gauge Silverman's needle. Each of the liver specimens included two consecutive sections; one was embedded in paraffin for hematoxylin and eosin staining, and the other was immediately preserved in liquid nitrogen until required for analysis. Only the 23 biopsy specimens confirmed to be hepatitis without dysplasia by histological examination were included in the hepatitis cohort.

Written approval was obtained from all patients before the initiation of this study. All protocols dealing with the patients conformed to the ethical guidelines of the Helsinki Declaration and were approved by Shandong University Research Ethics Committee.

\section{Immunohistochemical Staining}

Immunohistochemical studies were performed on serial paraffin sections encompassing both HCC and adjacent noncancerous liver tissue from each patient. The sections were deparaffinized and subjected to antigen retrieval by microwaving $(675 \mathrm{w})$ in $0.01 \mathrm{M}$ citrate buffer $(\mathrm{pH} 6.0)$ for $5 \mathrm{~min}$, followed by incubation in $3 \% \mathrm{H}_{2} \mathrm{O}_{2}$ for 10 min to quench endogenous peroxidases. Non-specific binding was blocked by incubating the sections in a blocking solution for $60 \mathrm{~min}$. Primary antibodies for IHC included specific antibodies against NLRP3 (no. ab17267, Abcam, Cambridge, MA, USA), ASC (no. ADI-905-173, Enzo life sciences, San Diego, CA, USA), IL-1 $\beta$ (no. ab2105, Abcam) and caspase 1 (no. AP6703c, Abgent, Shanghai, China). The sections were incubated overnight at $4{ }^{\circ} \mathrm{C}$ with primary antibodies diluted in $1 \%(\mathrm{wt} / \mathrm{vol})$ BSA. Substitution of the primary antibody with $1 \%$ BSA served as a negative control. The slides were washed with PBS three times and incubated with biotinylated secondary antibody at room temperature for $1 \mathrm{~h}$. The sections were stained by incubating the slides in 3,3'-diaminobenzidine, followed by counterstaining with hematoxylin. The sections were then dehydrated in alcohol and xylene, mounted in neutral gum, and analyzed using a bright field microscope.

\section{Evaluation of Immunohistochemical Staining}

Immunoreactivity was evaluated independently by two pathologists who were blinded with regard to the patient's clinical and pathological data. Six non-overlapping fields $(x 400)$ of view per biopsy were examined in a systematic 
a
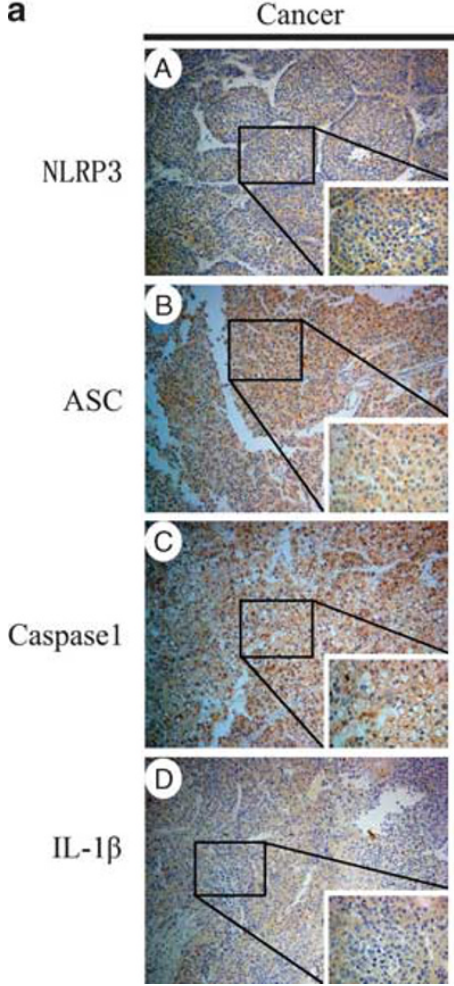

Non-Cancer
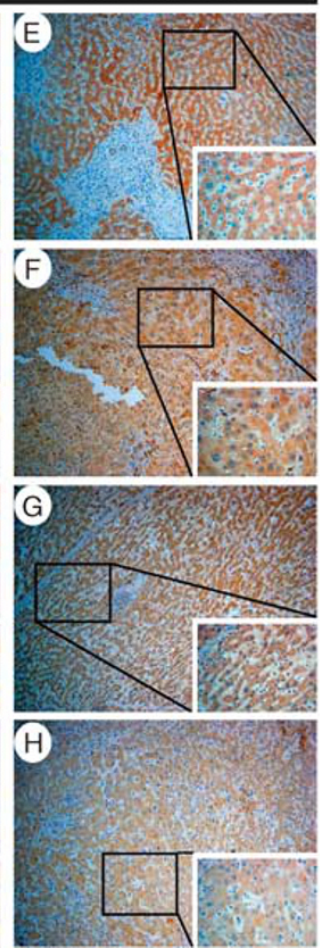

b Non-Cancer

Cancer

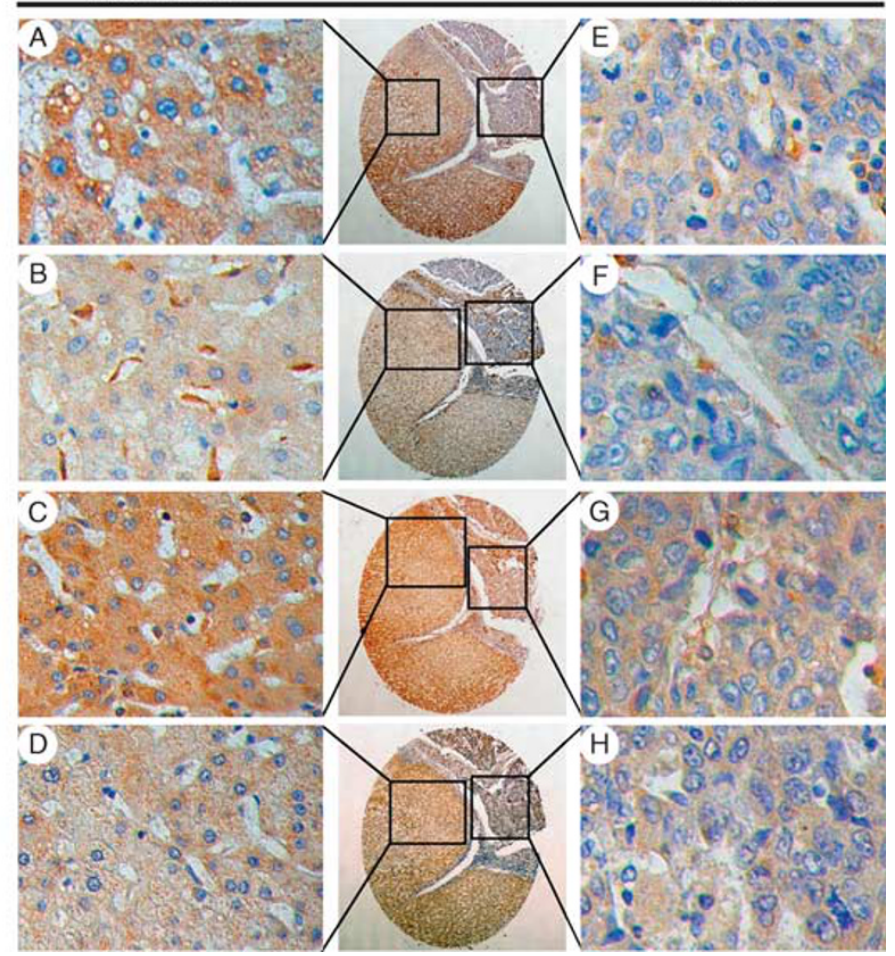

Figure 1 Expression of NLRP3 inflammasome components in HCC tissues and corresponding non-cancerous tissues by immunohistochemistry. (a) Staining of NLRP3, ASC, caspase 1, and interleukin (IL)-1 $\beta$ in hepatocellular carcinoma (HCC) tissues (A for NLRP3, B for ASC, C for caspase 1, and D for IL-1 $\beta$ ) and corresponding peritumoral liver tissues (E for NLRP3, F for ASC, G for caspase 1, and H for IL-1 $\beta$ ). (b) immunohistochemistry (IHC) staining of NLRP3, ASC, caspase 1, and IL-1 $\beta$ in a representative tissue core including both HCC tissues (A for NLRP3, B for ASC, C for caspase 1, and D for IL-1 $\beta$ ) and peritumoral non-cancerous liver tissues (E for NLRP3, F for ASC, G for caspase 1, and H for IL-1 $\beta$ ).

random sampling pattern. The intensity of staining was scored and graded on a $0-3$ scale, with no staining $=0$, weak staining $=1$, moderate staining $=2$, and strong staining $=3$. All of the cores were evaluated twice to confirm the reproducibility of the results. This initial score was measured as $0,1,2$, or 3 and further classified as low expression $(0-1)$ or high expression (2-3). In parallel, immunohistochemical staining was also evaluated using Image-Pro Plus v6.2 software (Media Cybernetics, Inc., Bethesda, MD, USA) as previously described. ${ }^{13}$ For accurate reading of the staining results for each antibody, we used the same setting for all the analyzed fields. Integrated optical density (IOD) was measured in each field, and positive staining density was formulated as IOD/the total area of each field.

\section{RT-PCR and Quantitative Real-Time PCR (qRT-PCR)}

Total RNA was extracted from HCC cells or fresh liver tissues using TRIzol reagent (Invitrogen, Carlsbad, CA, USA) according to the manufacturer's instructions. cDNA was synthesized from $2 \mu \mathrm{g}$ of total RNA using a Reverse Transcription Kit (Toyobo Co. Ltd., Osaka, Japan). Equal amounts of cDNA were subjected to PCR using the corresponding primers. The forward and reverse sequences of the primers can be provided upon request. PCR conditions included an initial denaturation at $94{ }^{\circ} \mathrm{C}$ for $5 \mathrm{~min}$, followed by 32 cycles of $94{ }^{\circ} \mathrm{C}$ for $30 \mathrm{~s}, 54^{\circ} \mathrm{C}$ for $30 \mathrm{~s}, 72^{\circ} \mathrm{C}$ for $30 \mathrm{~s}$, and a terminal extension at $72{ }^{\circ} \mathrm{C}$ for $5 \mathrm{~min}$. After the reaction, $10 \mu$ l of each PCR product was used for agarose gel electrophoresis. The gels were stained with ethidium bromide and visualized by UV transillumination. The qRT-PCR reaction was carried out using a Light Cycler (Bio-Rad, CFX96, USA) according to the manufacturer's instructions. The qRT-PCR amplification cycling conditions comprised an initial denaturation step at $95^{\circ} \mathrm{C}$ for $10 \mathrm{~min}$ and 40 PCR cycles of $95^{\circ} \mathrm{C}$ for $10 \mathrm{~s}$, $60{ }^{\circ} \mathrm{C}$ for $20 \mathrm{~s}$, and $72{ }^{\circ} \mathrm{C}$ for $15 \mathrm{~s}$. For each primer pair, we included a no-template control and a no-RT control (RTnegative) to verify that primer-dimer formation and genomic DNA contamination effects were negligible. Each sample was examined in triplicate, and $\beta$-actin was used as an internal control. PCR products were loaded in agarose gels and, in all cases, were confined to a single band of the expected size. A melting-curve analysis was performed to ensure the specificity of the products. The relative mRNA expression was determined using the comparative $\left(2^{-\Delta \Delta \mathrm{CT}}\right)$ method. GraphPad Prism v5 (GraphPad Software, Inc., San Diego, CA, USA) was used to create scatter plots. The data are representative of three independent experiments. 
Table 1 Clinicopathological characteristics of 128 patients with HCC

\begin{tabular}{|c|c|}
\hline Characteristics & No. of patients (\%) \\
\hline \multicolumn{2}{|l|}{ Gender } \\
\hline Male & $111(87.4 \%)$ \\
\hline Female & $17(13.4 \%)$ \\
\hline \multicolumn{2}{|l|}{ Age } \\
\hline$<54$ Years & $59(46.8 \%)$ \\
\hline$\geqslant 54$ Years & $67(53.2 \%)$ \\
\hline \multicolumn{2}{|l|}{ Tumor size } \\
\hline$<421 \mathrm{~cm}^{3}$ & $101(79.5 \%)$ \\
\hline$\geqslant 421 \mathrm{~cm}^{3}$ & $26(20.5 \%)$ \\
\hline \multicolumn{2}{|c|}{ Liver cirrhosis history } \\
\hline Yes & $88(68.8 \%)$ \\
\hline No & $40(31.2 \%)$ \\
\hline \multicolumn{2}{|l|}{ TNM stage } \\
\hline I & $61(48.0 \%)$ \\
\hline$\|$ & $32(25.2 \%)$ \\
\hline III & $25(19.7 \%)$ \\
\hline IV & $9(7.1 \%)$ \\
\hline \multicolumn{2}{|c|}{ Edmondson Grade } \\
\hline I & 17 (13.4\%) \\
\hline$\|$ & $81(63.8 \%)$ \\
\hline III & $36(28.3 \%)$ \\
\hline \multicolumn{2}{|c|}{ Regional lymph nodes } \\
\hline No & $125(97.7 \%)$ \\
\hline N1 & $2(2.3 \%)$ \\
\hline \multicolumn{2}{|c|}{ Distant metastasis } \\
\hline MO & $123(98.4 \%)$ \\
\hline M1 & $4(1.6 \%)$ \\
\hline
\end{tabular}

Abbreviation: HCC, hepatocellular carcinoma.

\section{Western Blot Analysis}

Total proteins were extracted from HCC cells or fresh liver tissues and lysed in RIPA buffer. Thereafter, $60 \mu \mathrm{g}$ of cell lysate was fractionated on $12 \%$ SDS-PAGE gels and transferred to polyvinylidene difluoride membranes. The membranes were probed with specific antibodies against NLRP3 (no. ALX-804-818-C100) and ASC (no. ADI-905-173) from
Table 2 Expression of the NLRP3 inflammasome components in HCC tissues and non-cancerous tissues

Feature $\quad$ No. of patients (\%)

\begin{tabular}{|c|c|c|}
\hline Cancerous tissues & Non-cancerous tissues & \\
\hline$\%$ & $\%$ & $\chi^{2}$ \\
\hline
\end{tabular}

NLRP3

\begin{tabular}{|c|c|c|c|c|c|c|}
\hline Low & 39 & 39.4 & 2 & 2.0 & & \\
\hline High & 60 & 60.6 & 97 & 98.0 & 42.000 & $0.000^{a}$ \\
\hline
\end{tabular}

ASC

$\begin{array}{lllllll}\text { Low } & 48 & 49.5 & 13 & 13.4 & & \\ \text { High } & 49 & 50.5 & 84 & 86.6 & 31.117 & \mathbf{0 . 0 0 0}^{\mathrm{a}}\end{array}$

Caspase 1

\begin{tabular}{crrrrrr} 
Low & 59 & 46.5 & 9 & 7.1 & & \\
High & 68 & 53.5 & 118 & 92.9 & 39.973 & $\mathbf{0 . 0 0 0 ^ { a }}$ \\
& & & & & & \\
IL-1 $\beta$ & & & & & & \\
Low & 57 & 61.3 & 4 & 4.3 & & \\
High & 36 & 38.7 & 89 & 95.7 & 74.051 & $\mathbf{0 . 0 0 0 ^ { a }}$ \\
\hline
\end{tabular}

Abbreviations: HCC, hepatocellular carcinoma; IL, interleukin. ${ }^{a} P<0.001$ by statistical analysis using Pearson's $\chi^{2}$-test.

Enzo Life Sciences and antibodies against caspase 1 (no. S3866) and IL-1 $\beta$ (no. S2022) from Cell Signaling Technology (Danvers, MA, USA). Expression of the $\beta$-actin protein was assessed as an internal control using anti- $\beta$-actin antibody (no. ab6276, Abcam).

\section{Statistical Analysis}

Data were analyzed using SPSS 16.0 (SPSS Inc., IL, USA) and expressed as the mean \pm s.e.m. $\chi^{2}$-tests were applied to compare categorical variables, and continuous variables were analyzed using the $t$-test. Spearman's rank correlation test was used to evaluate correlations between variables. A twotailed $P$-value was used in all analyses, and a $P$-value $<0.05$ was considered statistically significant.

\section{RESULTS}

NLRP3 Inflammasome Components were DownRegulated in Hepatic Parenchymal Cells in HCC Compared with Peritumoral Non-Cancerous Liver Tissues

Expression of NLRP3 inflammasome components was studied using IHC on 128 matched pairs of HCC tissues and corresponding peritumoral liver tissues. Positive staining for NLRP3, ASC, caspase 1 , and IL-1 $\beta$ was observed pre- 

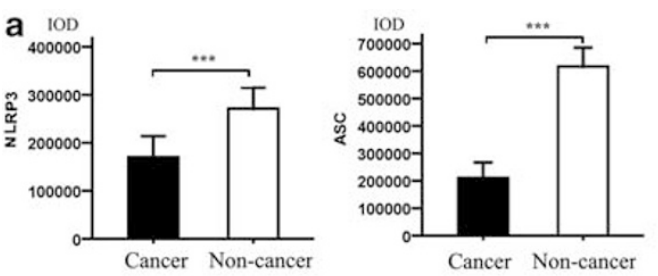

b
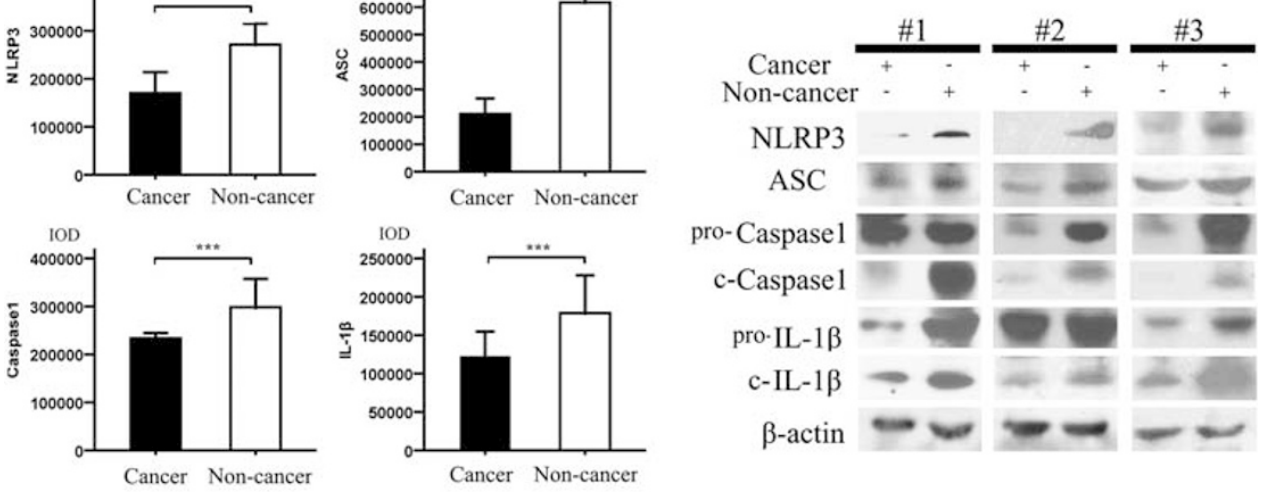

C
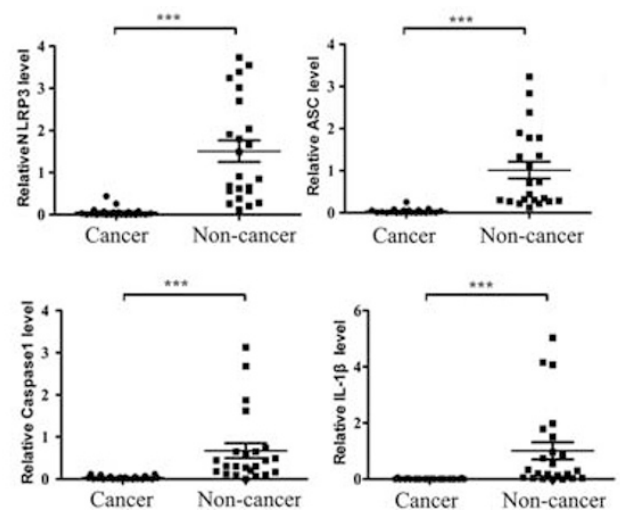

d

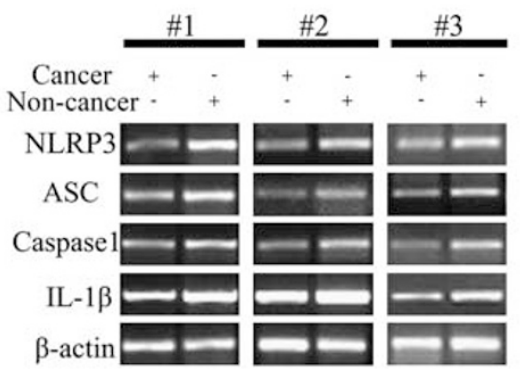

Figure 2 Quantitative analysis of NLRP3 inflammasome components expression in hepatocellular carcinoma (HCC) and peritumoral liver tissues. (a) Quantitative analysis of NLRP3 inflammasome expression in cancerous and non-cancerous liver tissues by Image-Pro Plus v6.2 software. (b) The protein expression levels of NLRP3, ASC, caspase 1, and interleukin (IL)-1 $\beta$ in HCC tissues and corresponding non-cancerous liver tissues were examined by western blot. The figures shown are the western blot bands from three representative cases. (c) mRNA expression levels of NLRP3, ASC, and caspase 1 were quantified by qRT-PCR in HCC tissues and paired non-cancerous liver tissues from 24 patients. (d) The mRNA expression levels of NLRP3 inflammasome components including NLRP3, ASC, caspase 1 , and IL-1 $\beta$ in HCC tissues and corresponding non-cancerous liver tissues were examined by RT-PCR. The presented bands are the RT-PCR products obtained from three representative cases. $(* * * P 0.001$ for all comparisons, HCC tissues vs peritumoral non-cancerous liver tissues).

dominantly in the cytoplasms of hepatocytes, HCC cells, bile duct epithelial cells, and macrophages, whereas specific immunoreactivity was negative in all infiltrating lymphocytes and neutrophils (Figure 1a). The negative control did not have any positive signal (data not shown), which confirmed that the positive staining resulted from staining with the specific antibody.

Immunoreactivity for the NLRP3 protein was significantly downregulated in HCC tissues compared with that of corresponding non-cancerous liver tissues $(P<0.001$, Table 2$)$. Moreover, consistent with the trend of NLRP3, the expression of other NLRP3 inflammasome components, including ASC, caspase 1, and IL- $1 \beta$ also showed deficient expression in HCC tissues compared with non-cancerous liver tissues $(P<0.001$, Table 2).

Typically, there were several cores including both the HCC and non-cancerous tissues within the same core, which showed contrasting expression of NLRP3, ASC, caspase 1, and IL- $1 \beta$ in the cancer and non-cancerous tissues. One of such typical cores is presented in Figure $1 b$.
To further confirm the downregulated expression of NLRP3 inflammasome components in HCC tissues, ImagePro Plus v6.2 was used to analyze immunoreactivity after staining the cancerous and non-cancerous tissues, and the data are shown in Figure 2a. The results further confirmed the significant downregulation of NLRP3 inflammasome components in HCC tissues.

Validation of the expression tendency of NLRP3 inflammasome components was further performed by RT-PCR, qRT-PCR, and western blot analyses to detect the mRNA and protein levels of NLRP3 inflammasome components in fresh HCC tissues and corresponding non-cancerous liver tissues from another cohort of 24 HCC patients. Western blot analysis confirmed the decreased expression of NLRP3 inflammasome proteins in cancerous tissues compared with that in the corresponding non-cancerous tissues. Representative immunoblot bands from three HCC patients are shown in Figure 2b. The mRNA expression of NLRP3 inflammasome components was examined by RT-PCR and qRT-PCR. The qRT-PCR and RT-PCR analyses confirmed 
Table 3 Correlations among the NLRP3 inflammasome components in HCC tissues

\begin{tabular}{|c|c|c|c|c|c|c|c|c|c|c|c|c|c|c|c|c|c|c|}
\hline \multirow[b]{2}{*}{ IL-1 $\beta$} & \multicolumn{6}{|c|}{ NLRP3 } & \multicolumn{5}{|c|}{ ASC } & \multicolumn{7}{|c|}{ Caspase 1} \\
\hline & - & + & ++ & +++ & $R$ & $P$ & - & + & ++ & $R$ & $P$ & - & + & ++ & & & $R$ & $P$ \\
\hline- & 32 & 8 & 8 & 0 & & & 34 & 8 & 6 & & & 28 & 16 & 3 & & & & \\
\hline+ & 4 & 40 & 6 & 0 & & & 15 & 30 & 6 & & & 20 & 15 & 14 & & & & \\
\hline++ & 4 & 1 & 4 & 1 & & & 1 & 5 & 3 & & & 4 & 4 & 1 & & & & \\
\hline \multirow[t]{2}{*}{+++} & 0 & 4 & 0 & 0 & 0.398 & $0.000^{\mathrm{a}}$ & 0 & 0 & 4 & 0.455 & $0.000^{\mathrm{a}}$ & 1 & 1 & 2 & & & 0.224 & $0.017^{b}$ \\
\hline & \multicolumn{6}{|c|}{ NLRP3 } & \multicolumn{5}{|c|}{ ASC } & \multicolumn{7}{|c|}{ NLRP3 } \\
\hline Caspase 1 & - & + & ++ & +++ & $R$ & $P$ & - & + & ++ & $R$ & $P$ & ASC & - & + & ++ & +++ & $R$ & $P$ \\
\hline- & 27 & 21 & 5 & 0 & & & 31 & 20 & 2 & & & - & 31 & 16 & 3 & 0 & & \\
\hline+ & 12 & 15 & 9 & 0 & & & 11 & 15 & 10 & & & + & 5 & 28 & 10 & 0 & & \\
\hline++ & 1 & 16 & 3 & 0 & & & 8 & 7 & 5 & & & ++ & 4 & 9 & 5 & 1 & & \\
\hline+++ & 0 & 1 & 1 & 1 & 0.354 & $0.000^{\mathrm{a}}$ & 0 & 1 & 2 & 0.318 & $0.001^{b}$ & +++ & 40 & 53 & 18 & 1 & 0.454 & $0.000^{\mathrm{a}}$ \\
\hline
\end{tabular}

Abbreviation: HCC, hepatocellular carcinoma.

$R$ : Spearman's correlation coefficient.

${ }^{\mathrm{a}} P<0.001$ by statistical analysis using Spearman's rank correlation test.

${ }^{\mathrm{b}} P<0.05$. 
a

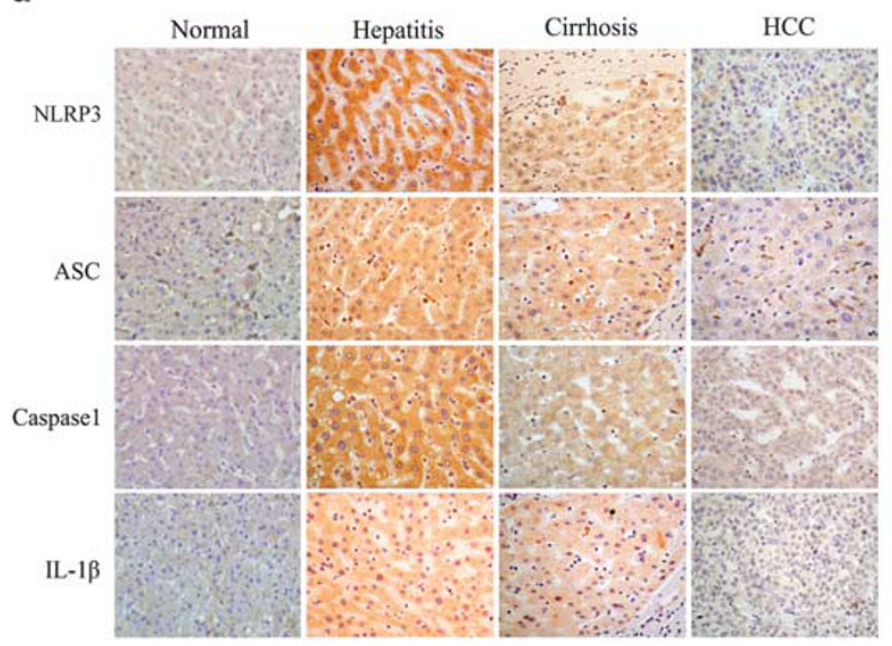

b
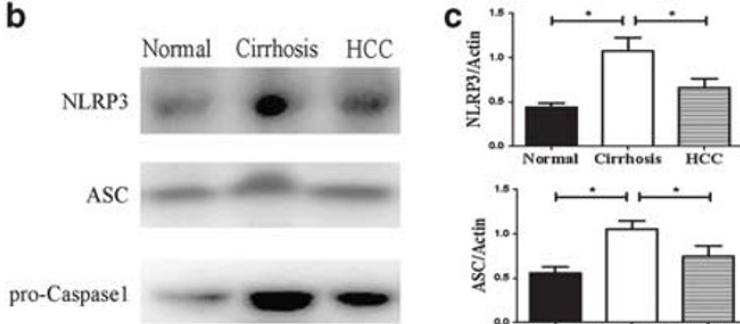

pro-Caspase l
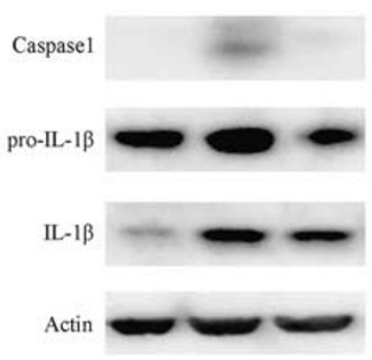
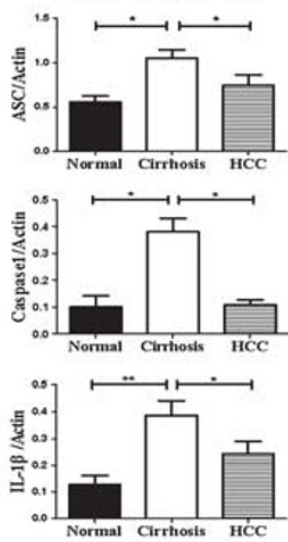

d

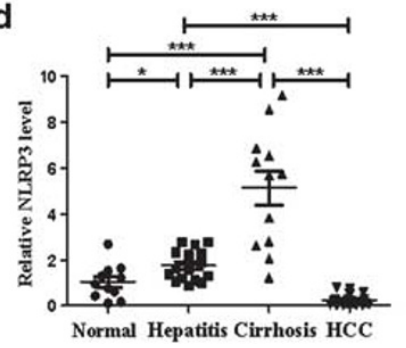

e

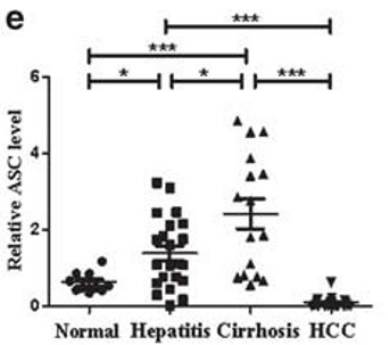

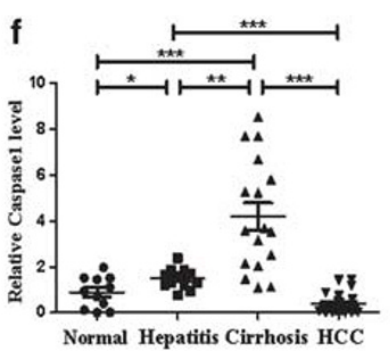

Figure 3 Expression of NLRP3 inflammasome components during multistage carcinogenesis. (a) Expression of NLRP3, ASC, caspase 1, and interleukin (IL)-1 $\beta$ in normal liver, peritumoral hepatitis and cirrhotic tissues, and hepatocellular carcinoma (HCC) tissues was detected by immunohistochemistry (IHC) staining. (b, c) Expression of the NLRP3 inflammasome components in normal liver tissues, cirrhotic tissues, and HCC tissues was done by western blot. The presented figure includes representative blots showing the expression levels in different specimens (b). The band intensity analysis is shown in the histogram and was statistically analyzed (c). (d- $\mathbf{g})$ The mRNA expression levels of NLRP3 (d), ASC (e), caspase 1 (f), and IL-1 $\beta$ (g) in fresh normal liver, hepatitis, cirrhosis, and HCC tissues were examined by qRT-PCR. $\left({ }^{*} P<0.05,{ }^{* *} P<0.01,{ }^{* * *} P<0.001\right)$.

that NLRP3 inflammasome components had significantly decreased mRNA expression in liver cancer tissues when compared with the corresponding non-cancerous tissues (Figures $2 \mathrm{c}$ and $\mathrm{d}$ ). The representative bands of the RT-PCR products of the cancerous and corresponding non-cancerous liver tissues were obtained from three HCC patients. All of these data validated the results of the IHC analysis and further confirmed the decreased expression tendency in liver cancer tissues compared with non-cancerous tissues.

\section{The Expression Levels of the NLRP3 Inflammasome Components had Significantly Positive Correlations with One Another}

Statistical analysis of the IHC scores showed that the expression levels of NLRP3, ASC, caspase 1 , and IL- $1 \beta$ had well-defined positive correlations with one another $(P<0.05$, Table 3). These results suggested that NLRP3, ASC, caspase 1, and IL-1 $\beta$ constituted a multiprotein NLRP3 inflammasome platform and cooperated to participate in HCC development.

\section{The Expression Pattern of the NLRP3 Inflammasome was Further Investigated in Multi-Stage Hepatocarcinogenesis}

HCC nearly always develops in the setting of chronic hepatitis or cirrhosis. To elucidate the role of the multiprotein NLRP3 inflammasome platform in the process of hepatocarcinogenesis, we analyzed its expression in normal liver tissues, peritumoral hepatitis and cirrhotic tissues, and corresponding HCC tissues, which represented multi-stage hepatocarcinogenesis. Images of the typical IHC staining patterns of the NLRP3 inflammasome components in this process are presented in Figure 3a. Statistical analysis revealed that expression of all of the NLRP3 inflammasome components was significantly downregulated in HCC tissues compared with the surrounding hepatitis and cirrhotic tissues $(P<0.001$, Table 4$)$. However, there was no significant difference between the normal liver tissue and the liver cancer tissue. These data suggested that NLRP3 inflammasome expression in normal liver tissue was low; that expression during liver injury, including hepatitis and cirrhosis, was upregulated; and that expression was significantly down- 
Table 4 Expression of NLRP3 inflammasome components in HCC and peritumoral hepatitis/cirrhosis tissues

\begin{tabular}{|c|c|c|c|c|c|c|}
\hline & \multicolumn{3}{|c|}{ Liver tissues } & \multicolumn{3}{|c|}{ Liver tissues } \\
\hline & Hepatitis & $\mathrm{HCC}$ & $P$ & Cirrhosis & $\mathrm{HCC}$ & $P$ \\
\hline \multicolumn{7}{|l|}{ NLRP3 } \\
\hline Negative & 6 & 92 & & 9 & 92 & \\
\hline Positive & 50 & 20 & $0.000^{\mathrm{a}}$ & 47 & 20 & $0.000^{\mathrm{a}}$ \\
\hline \multicolumn{7}{|l|}{ ASC } \\
\hline Negative & 27 & 86 & & 30 & 86 & \\
\hline Positive & 28 & 27 & $0.000^{\mathrm{a}}$ & 23 & 27 & $0.000^{\mathrm{a}}$ \\
\hline \multicolumn{7}{|l|}{ Caspase 1} \\
\hline Negative & 19 & 92 & & 25 & 92 & \\
\hline Positive & 41 & 35 & $0.000^{\mathrm{a}}$ & 44 & 35 & $0.000^{\mathrm{a}}$ \\
\hline \multicolumn{7}{|l|}{$I L-1 \beta$} \\
\hline Negative & 24 & 91 & & 13 & 91 & \\
\hline Positive & 30 & 21 & $0.000^{\mathrm{a}}$ & 37 & 21 & $0.000^{\mathrm{a}}$ \\
\hline
\end{tabular}

Abbreviations: HCC, hepatocellular carcinoma; IL, interleukin. ${ }^{\text {a }} P<0.001$ by statistical analysis using Pearson's $\chi^{2}$-test. regulated when hepatocarcinoma developed. The expression tendency of the inflammasome components at the protein level was confirmed by western blot analysis of fresh liver tissues. Because of the limited amount of hepatitis specimens, the western blot analysis was performed using normal liver tissues, cirrhotic tissues and liver cancer tissues, and representative blot bands are presented and analyzed (Figures $3 \mathrm{~b}$ and $\mathrm{c}$ ). The results confirmed that NLRP3 inflammasome expression was low in normal liver tissues, upregulated in cirrhotic liver tissues, and significantly downregulated in liver cancer tissues.

To determine whether the expression tendency was also present in the mRNA levels of the NLRP3 inflammasome components, we performed qRT-PCR analysis on four panels of liver tissues, including 9 normal liver controls, 23 hepatitis biopsy tissues, 24 cirrhotic tissues, and 24 HCC tissues. As shown in Figures $3 \mathrm{~d}-\mathrm{g}$, qRT-PCR analysis confirmed the tendency of these NLRP3 inflammasome components to change during multi-stage carcinogenesis; compared with the basal levels of expression in normal liver tissues, expression was upregulated in inflammatory liver diseases, including hepatitis and cirrhosis, and was significantly downregulated in liver cancer tissues. Altogether, these data further confirmed our previously observed NLRP3 inflammasome expression pattern in hepatocarcinogenesis. a
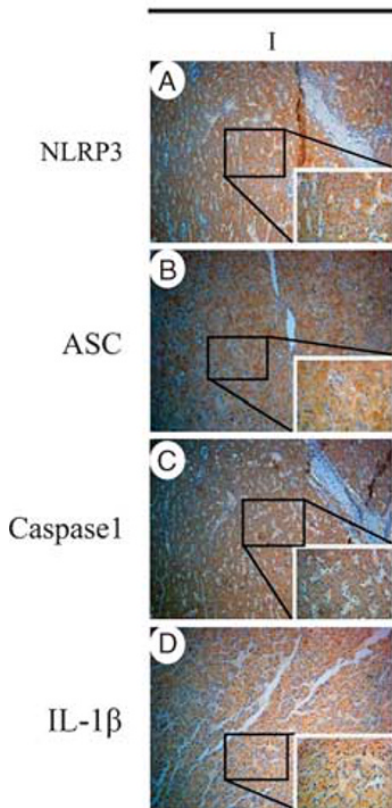

IHC
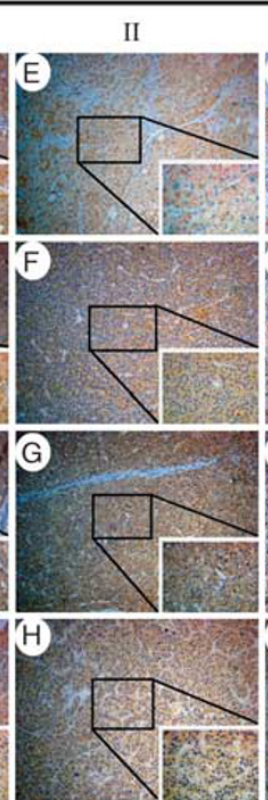
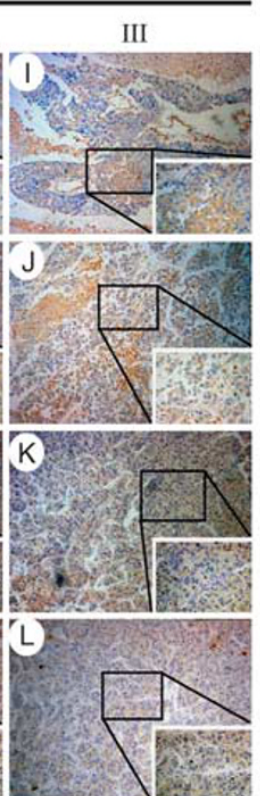

b
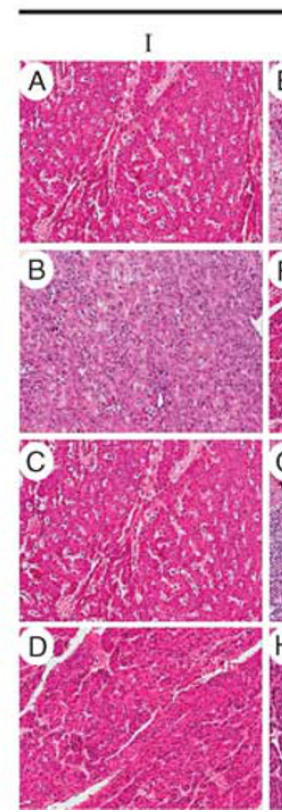

HE
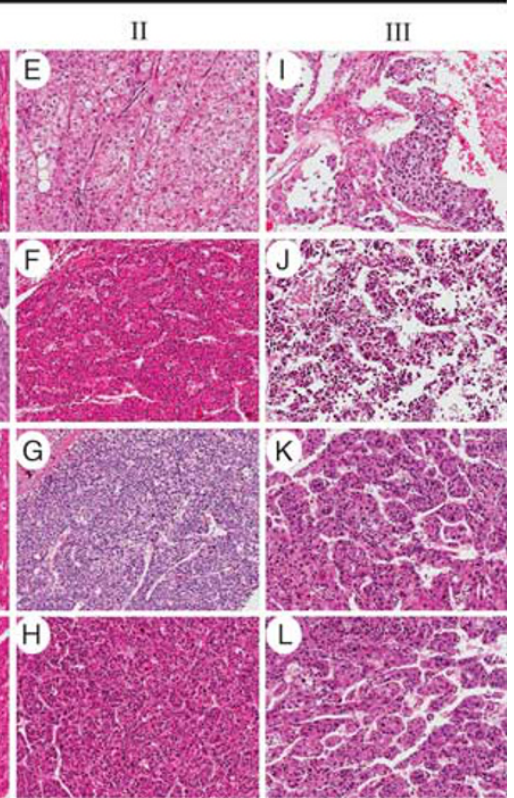

Figure 4 Immunohistochemistry (IHC) and hematoxylin and eosin (H\&E) staining for NLRP3, ASC, caspase 1, and interleukin (IL)-1 $\beta$ in hepatocellular carcinoma (HCC) tissues with different Edmonson grades. (a) Photograph of IHC for NLRP3, ASC, caspase 1, and IL-1 $\beta$ in Edmonson Grade I (A for NLRP3, B for ASC, C for caspase 1, and D for IL-1 $\beta$ ), Grade II (E for NLRP3, F for ASC, G for caspase 1, and H for IL-1 $\beta$ ), and Grade III (I for NLRP3, J for ASC, $\mathrm{K}$ for caspase 1, and L for IL-1 $\beta$ ) liver cancer tissues. (b) HE staining of the same cores to identify Edmonson Grade I (A for NLRP3, B for ASC, C for caspase 1, and D for IL-1 $\beta$ ), Grade II (E for NLRP3, F for ASC, G for caspase 1, and H for IL-1 $\beta$ ), and Grade III (I for NLRP3, J for ASC, K for caspase 1, and $\mathrm{L}$ for IL-1 $\beta$ ) HCC tissues. 
Table 5 Correlations between NLRP3, ASC, caspase 1, and IL-1 $\beta$ expression levels in HCC tissues and clinicopathologic features

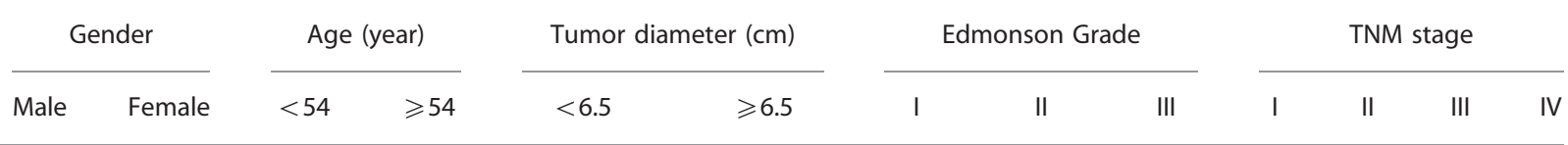

NLRP3

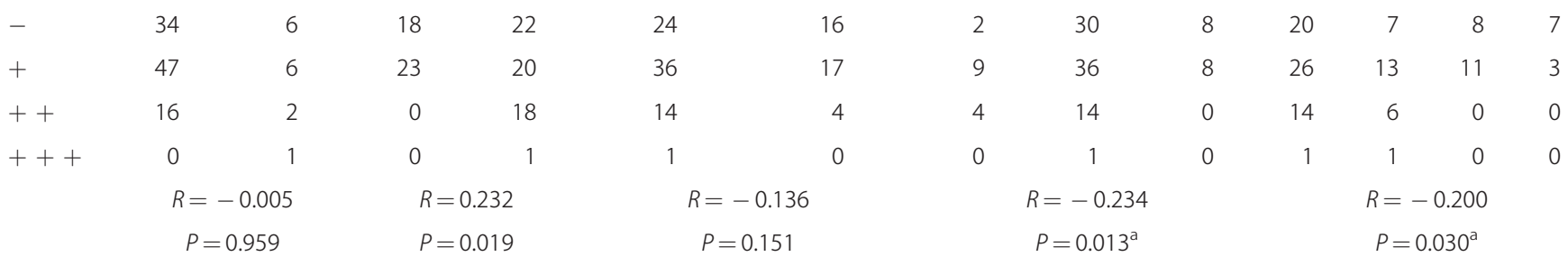

ASC

\begin{tabular}{|c|c|c|c|c|c|c|c|c|c|c|}
\hline- & 44 & 26 & 24 & 27 & 6 & 31 & 12 & 24 & 8 & 12 \\
\hline+ & 37 & 16 & 34 & 9 & 6 & 37 & 3 & 22 & 17 & 3 \\
\hline++ & 16 & 10 & 17 & 2 & 3 & 14 & 1 & 12 & 7 & 0 \\
\hline & $R=0.017$ & $R=0.047$ & \multicolumn{2}{|c|}{$R=-0.373$} & \multicolumn{3}{|c|}{$R=-0.188$} & & \multicolumn{2}{|c|}{$R=-0.221$} \\
\hline & $P=0.859$ & $P=0.619$ & \multicolumn{2}{|c|}{$P=0.000^{\mathrm{a}}$} & \multicolumn{3}{|c|}{$P=0.046^{\mathrm{a}}$} & & \multicolumn{2}{|c|}{$P=0.019^{a}$} \\
\hline
\end{tabular}

Caspase 1

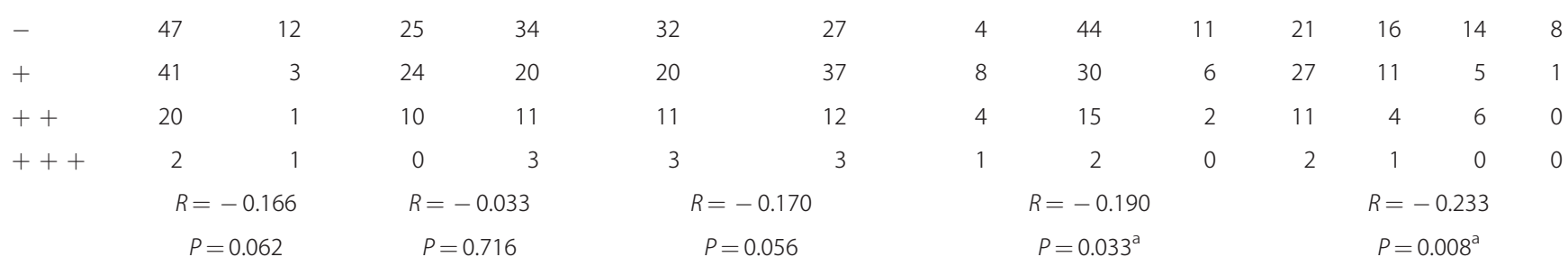

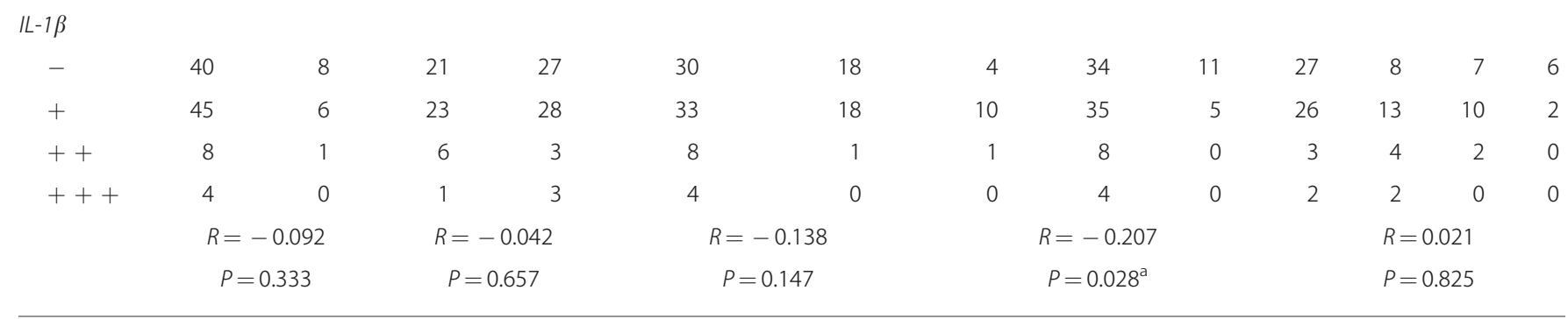

Abbreviations: HCC, hepatocellular carcinoma; IL, interleukin.

${ }^{a} P<0.05$ by statistical analysis using Spearman's rank correlation test.

Loss of the NLRP3 Inflammasome was Correlated with a Higher HCC Pathological Grade and More Advanced Clinical Stages in HCC Patients

IHC indicated that patients with poorer pathological differentiation of HCC were prone to have weaker immunoreactivity to the NLRP3 inflammasome components, whereas patients with a more advanced disease stage were likely to have lower NLRP3 inflammasome expression (Figure 4). To further elucidate the potential link between expression of the NLRP3 components and clinicopathological features, a correlation analysis was applied to these data. The analysis showed that the expression levels of the NLRP3 inflammasome components were inversely correlated with pathological grades and clinical stages in the HCC patients $(P<0.05$, Table 5). This indicated that loss of the NLRP3 inflammasome was involved in HCC progression, which indicated its protective role in the development of cancer.

\section{DISCUSSION}

HCC is a primary malignancy of the liver and the third leading cause of cancer death. ${ }^{6}$ It frequently develops in the context of chronic hepatitis and cirrhosis, characterized by 
hepatic inflammation, fibrosis, and apoptosis. In this study, we conducted a comprehensive analysis of the expression of NLRP3 inflammasome components in the parenchymal cells of normal liver, non-cancerous inflammatory liver, and liver cancer tissues, which represented the typical progression of multi-stage hepatocarcinogenesis. Our data showed that inflammasome expression was relatively low in normal hepatocytes and that it was significantly upregulated in the inflammatory hepatic setting and significantly downregulated in malignantly transformed liver cancer tissues.

This dynamic expression pattern offered a clue for the full understanding of the pathogenesis of chronic liver diseases, and it may be explained by the functional role of the inflammasome as a guardian of the sanctity of the intracellular cytosol. When the body is exposed to a pathogenic insult, the NLRP3 inflammasome acts as a guard and is assembled and activated to elicit hepatic inflammation and promote the reestablishment of homeostasis. However, chronic persistent inflammation and inflammasome activation induce massive cell death, which are healed by fibrosis, compensatory hepatocyte proliferation, and liver regeneration. Thus, hepatocytes proliferating under conditions of continuous liver injury may accumulate mutations, leading to dysplasia and, eventually, to tumor development. Once a hepatocarcinoma formed, the expression of the NLRP3 inflammasome components was significantly downregulated in liver cancer cells, indicating that malignant parenchymal cells originate from NLRP3 inflammasome-deficient precursor cells.

The significant downregulation of the NLRP3 inflammasome in malignantly transformed parenchymal cells indicated the loss of the protective role of the NLRP3 inflammasome. Our data revealed that HCC patients with lower expression of NLRP3 inflammasome components were prone to have poorer cancer cell differentiation and more advanced clinical stages, indicating that downregulation of this NLRP3 inflammasome molecular platform is involved in HCC progression. Although the active involvement of the NLRP3 inflammasome in liver injury has been reported, ${ }^{14,15}$ the involvement of its significant downregulation in malignantly transformed liver cancer cells is reported for the first time in this study. Our seemingly counterintuitive data are logical considering the diverse roles of the NLRP3 inflammasome. ${ }^{4,16}$ On one hand, in addition to the classic function of the inflammasome in detecting danger signals and causing inflammation, another alternative function of the inflammasome is to dictate cell fate, as significant activation of the inflammasome can lead to cell death. , $^{217,18}$ HCC typically originates from hepatic inflammation, and intense and chronic inflammatory responses are almost invariably accompanied by a massive loss of hepatocytes and irreversible damage to the liver. Dying hepatocytes release damage-associated molecular patterns, which aggravate inflammation and further damage the liver. ${ }^{19}$ Thus, a positive feedback loop is formed, amplifying the cycle of cell death and compensatory liver regeneration, which further increases the risk of liver cancer. On the other hand, the NLRP3 inflammasome is a danger sensor, and loss of its expression may result in the ignorance of danger signals present in the cytoplasm and compromise cell survival with mistakes. ${ }^{3,16,20,21}$ As a consequence, the accumulation of damage, mistakes, and mutations results in cancer. Furthermore, cell immortality and inappropriate cell survival resulting from NLRP3 inflammasome deregulation confers resistance to cancer therapy, which may explain the resistance of HCC to a variety of anticancer drugs used in liver cancer patients.

Our data, which demonstrated that a deficiency in inflammasome expression was correlated with cancer progression, parallel previous findings in colon cancer, which suggest that the NLRP3 inflammasome has a protective role against the development of cancer. ${ }^{12,20,22,23}$ However, the data are in disagreement with several reports stating that high IL-1 $\beta$ secretion in the tumor microenvironment is correlated with a more malignant phenotype, indicating that these inflammasome molecules may have a role in the promotion of cancer development. ${ }^{24,25}$ However, these seemingly opposite reports regarding the roles of the NLRP3 inflammasome in cancer are not controversial, considering the pleiotropic and sometimes contrasting roles of the NLRP3 inflammasome in the multiple facets of oncogenesis. On one hand, inflammasome activation operates at the cell-autonomous level to eliminate malignant precursors through the induction of cell death. ${ }^{2,17,18}$ On the other hand, the cleavage and release of the inflammasome effector molecule IL-1 $\beta$ may fuel the inflammatory cycle in the cancer microenvironment that leads to sterile inflammation and carcinogenesis. ${ }^{26}$ Given the complex nature of the NLRP3 inflammasome, its complicated and even contrasting roles in cancer are understandable. Nevertheless, these divergently reported roles of the NLRP3 inflammasome in cancer may also be the result of different experimental systems and different methodologies. Most of the investigations of the inflammasome have been conducted using genetically modified animal models rather than clinical cohorts. To our knowledge, this is the first study to investigate the role of the NLRP3 inflammasome in carcinogenesis in the clinic in cancer patients. These data, which were directly gathered from clinical patients, represent actual pathological situations in vivo and are more convincing than animal models.

In summary, this study provided a comprehensive investigation of the multiprotein NLRP3 inflammasome platform in the parenchymal cells of HCC tissues. Our data revealed a dynamic expression pattern of NLRP3 inflammasome components in the development of HCC and demonstrated that a deficiency of this multiprotein platform was involved in HCC progression.

\section{ACKNOWLEDGMENTS}

This study was supported by the National Nature Science Foundation of China (no. 81172352 and no. 30700357) and the Science and Technology Development Project of Shandong Province (no. 2011GGE27020). 


\section{DISCLOSURE/CONFLICT OF INTEREST}

The authors declare no conflict of interest.

1. Church LD, Cook GP, McDermott MF. Primer: inflammasomes and interleukin 1 beta in inflammatory disorders. Nat Clin Pract Rheumatol 2008;4:34-42.

2. Strowig T, Henao-Mejia J, Elinav E, et al. Inflammasomes in health and disease. Nature 2012;481:278-286.

3. Martinon F, Mayor A, Tschopp J. The inflammasomes: guardians of the body. Annu Rev Immunol 2009;27:229-265.

4. Petrilli V, Dostert C, Muruve DA, et al. The inflammasome: a danger sensing complex triggering innate immunity. Curr Opin Immunol 2007;19:615-622.

5. Ogura Y, Sutterwala FS, Flavell RA. The inflammasome: first line of the immune response to cell stress. Cell 2006;126:659-662.

6. Block TM, Mehta AS, Fimmel CJ, et al. Molecular viral oncology of hepatocellular carcinoma. Oncogene 2003;22:5093-5107.

7. Yazdi AS, Drexler SK, Tschopp J. The role of the inflammasome in nonmyeloid cells. J Clin Immunol 2010;30:623-627.

8. Peeters PM, Perkins TN, Wouters EF, et al. Silica induces NLRP3 inflammasome activation in human lung epithelial cells. Part Fibre Toxicol 2013;10:3

9. Luedde T, Beraza N, Kotsikoris V, et al. Deletion of NEMO/IKKgamma in liver parenchymal cells causes steatohepatitis and hepatocellular carcinoma. Cancer Cell 2007;11:119-132.

10. Lockwood DS, Yeadon TM, Clouston AD, et al. Tumor progression in hepatocellular carcinoma: relationship with tumor stroma and parenchymal disease. J Gastroenterol Hepatol 2003;18: 666-672.

11. Zaki MH, Boyd KL, Vogel $\mathrm{P}$, et al. The NLRP3 inflammasome protects against loss of epithelial integrity and mortality during experimental colitis. Immunity 2010;32:379-391.

12. Allen IC, TeKippe EM, Woodford RM, et al. The NLRP3 inflammasome functions as a negative regulator of tumorigenesis during colitisassociated cancer. J Exp Med 2010;207:1045-1056.
13. Li T, Zhu Y, Qin CY, et al. Expression and prognostic significance of vascular endothelial growth factor receptor 1 in hepatocellular carcinoma. J Clin Pathol 2012;65:808-814.

14. Ganz M, Csak T, Nath B, et al. Lipopolysaccharide induces and activates the Nalp3 inflammasome in the liver. World J Gastroenterol 2011;17:4772-4778.

15. Imaeda $A B$, Watanabe $A$, Sohail $M A$, et al. Acetaminophen-induced hepatotoxicity in mice is dependent on Tlr9 and the Nalp3 inflammasome. J Clin Invest 2009;119:305-314.

16. Martinon F. Detection of immune danger signals by NALP3. J Leukoc Biol 2008;83:507-511.

17. Schroder K, Tschopp J. The inflammasomes. Cell 2010;140:821-832.

18. Franchi L, Eigenbrod T, Munoz-Planillo R, et al. The inflammasome: a caspase-1-activation platform that regulates immune responses and disease pathogenesis. Nat Immunol 2009;10:241-247.

19. Brenner C, Galluzzi L, Kepp O, et al. Decoding cell death signals in liver inflammation. J Hepatol 2013.

20. Anthony K. Immunology: the inflammasome protects? Nat Rev Cancer 2010;10:383.

21. Kanneganti TD, Ozoren N, Body-Malapel M, et al. Bacterial RNA and small antiviral compounds activate caspase-1 through cryopyrin/ Nalp3. Nature 2006;440:233-236.

22. Zaki MH, Lamkanfi M, Kanneganti TD. The Nlrp3 inflammasome: contributions to intestinal homeostasis. Trends Immunol 2011;32:171-179.

23. Zaki MH, Vogel P, Body-Malapel M, et al. IL-18 production downstream of the Nlrp3 inflammasome confers protection against colorectal tumor formation. J Immunol 2010;185:4912-4920.

24. Okamoto M, Liu W, Luo Y, et al. Constitutively active inflammasome in human melanoma cells mediating autoinflammation via caspase- 1 processing and secretion of interleukin-1beta. J Biol Chem 2010;285:6477-6488.

25. Hu B, Elinav E, Huber $\mathrm{S}$, et al. Inflammation-induced tumorigenesis in the colon is regulated by caspase-1 and NLRC4. Proc Natl Acad Sci USA 2010;107:21635-21640.

26. Zitvogel L, Kepp O, Galluzzi L, et al. Inflammasomes in carcinogenesis and anticancer immune responses. Nat Immunol 2012;13:343-351. 